\title{
Public-private partnership approach to non- communicable diseases prevention in Ghana
}

\begin{abstract}
The increasing prevalence of Non-Communicable Diseases (NCDs) related deaths in Ghana have become a general concern for both researchers and policy actors. As a measure to lowering NCDs deaths incidence, this paper provides an integrated approach to NCDs prevention emphasizing the need for a public-private approach to fighting this health menace. The review indicates that a consensual goal-oriented actions and guidelines between international agencies, state actors and Coalition of Non-governmental organization for Health (CNGO) is the drive to eradicating the incidence of NCDs in Ghana. In conclusion, the paper advocate that PPP approach should be built on a multicultural and resilient action plans in order to win the fight against the high prevalence of NCDs which is inadvertently causing high level of mortality in Ghana and beyond.
\end{abstract}

Keywords: public-private partnership, non-communicable diseases, Ghana, incidence
Volume 6 Issue 3 - 2017

Kofi Aduo-Adjei

Institute of Demography, National Research University, Russia

Correspondence: Kofi Aduo-Adjei, Institute of Demography, National Research University, Higher School of Economics, Moscow, Russia, Tel +79295724584, Email aduoadjei@gmail.com

Received: August 09, 2017| Published: September 20, 2017
Abbreviations: NCD, non-communicable diseases; SSA, sub saharan africa; WHO, world health organization; PPP, public-private partnership; NHIS, national health insurance scheme

\section{Introduction}

Global mortality statistics keep increasing at an alarming rate. It is established by World Health Organization (WHO) the growing contribution of Non-Communicable Diseases (NCDs) to the global disease burden and mortality incidence. ${ }^{1}$ Evidence indicates that NCDs are poignantly responsible for approximately $63 \%$ of the 57 million deaths that occurred in 2015 as these deaths were linked with cancers, diabetes, cardiovascular illness coupled with other respiratory diseases. ${ }^{1,2}$ Evidently, there is a highest burden of morbidity and mortality in middle and high-income countries resulting from NCDs, with significant proportions in developing economies, essentially in Sub-Saharan Africa (SSA). It was estimated that one-fourth of deaths occurring in SSA was accounted by Non-Communicable Diseases. ${ }^{3} \mathrm{~A}$ striking projection by WHO reveals that death caused by NCDs will increase to $60 \%$ from 2020 to $2030 .{ }^{4}$ This has both population and public health implications for African countries in promoting their quality of health, a desired target for the United Nations Sustainable Development Goal on Health (SDG, Goal 3).

\section{The Ghana context - NCDs}

Empirical evidence suggests an increasing share of Ghana's burden of NCDs, which have been described as worrying by researchers and policymakers alike. It is noted that NCDs accounts for $39 \%$ of all deaths occurring in $2015 .{ }^{4}$ In these deaths WHO estimates that cardiovascular diseases contribute to $18 \%$, with cancers $(6 \%)$, respiratory diseases and diabetes constituting $5 \%$ and $1 \%$ respectively. ${ }^{4-6}$ The increasing prevalence of NCD in Ghana is associated with improved life expectancy, as NCDs incidence is highly associated with survival through middle to adult ages. ${ }^{7}$ Again, evidence reveals that lifestyle and behavioral changes resulting from rapid urbanization and globalization as well as poor dietary habits due to excessive work pressures predisposes most Ghanaians to NCDs in a face of increasing physical inactivity and sedentary lifestyles. In the wake of fighting the surge of NCDs in Ghana, a body of research have excessively argued, the burden of NCDs emphasizing households and children burden as well as socio-economic determinants and theoretical approaches to prevalence of NCDs in Ghana. However, in the quest of finding an effective intervention to NCDs, there is the need to move beyond biomedical and epidemiological studies. ${ }^{8-10}$ Thus, embracing the actors and stakeholder's commitment to the fight against prevalence of NCDs, thereby addressing public-private partnership engagement to NCDs prevention is pivotal in dealing with global diseases management and cure.

\section{Public-private partnership integrated approach to NCDs}

The failure of state and market actors to promote and uphold health care as a public good has resulted in the emergence of public-private partnership (PPP) paradigm as a form of global health governance. The fulcrum of this engagement is to stimulate the maximum cooperation of private sector, international agencies, and non-governmental organization in collaboration with government in addressing health inequalities and challenges. Evidence indicates a successful partnership of both state and private actors in this partnership framework against preventing NCDs in advanced economies. Reflection on the evidence of PPP among the developed nations paves a strong footing for Ghana in NCDs prevention. ${ }^{6,7}$ The coalition of NGOs for Health $(\mathrm{CNGOH})$ working in tandem with the state actors, i.e Ghana Health Services, Ministerial of Health and other parastatal institution promotes a common defined goal to tackling and preventing NCDs. This effort can be results yielding with support from international organizations in providing policy and technical advisory services. Since a wellarticulated policy guideline will propel a formidable action plan for the fight against NCDs in Ghana. 


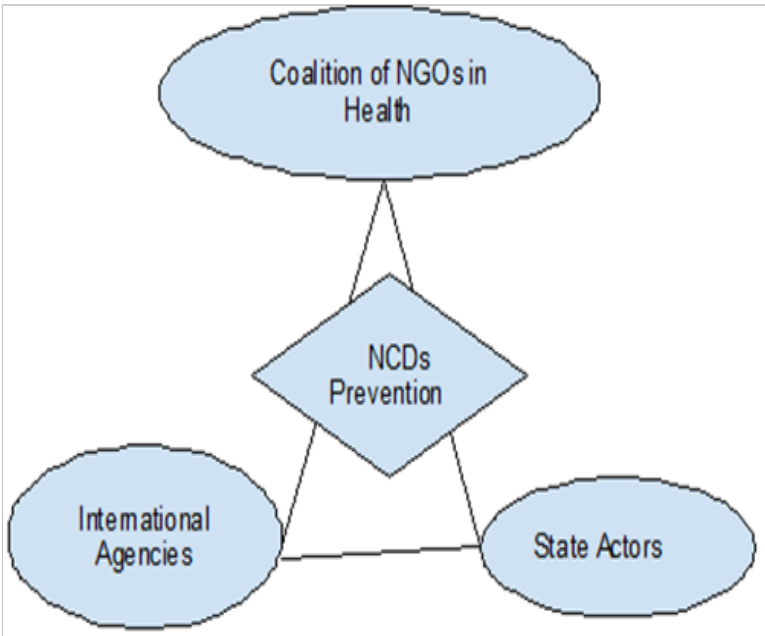

Figure I NCDs prevention partnership framework.

\section{Winning the fight against non-communicable disease (NCD)}

Essentially, government and private stakeholders need a common shared vision with a defined target to guide their engagement to advocacy and prevention of NCDs. A commitment denudes of different values, interests and worldviews is critical in resolving and preventing the prevalence of Non-Communicable Diseases in Ghana. Furthermore, one cannot be silent about the need for sustainable health financing to NCDs through the National Health Insurance Scheme in Ghana (NHIS) ${ }^{8,9}$ Thus, financially resourcing the primary health care providers in patients centered service provision to NCDs patients have strong reflection on the policy aim of Universal Health Coverage (UHC), which has a global tone of making healthcare accessible and equitable irrespective of the management and cure cost of the diseases. ${ }^{10-12}$

\section{Conclusion}

In summary, achieving quality health and promoting wellbeing for all is the desired goal that requires total commitment of government and the private sector in policy and practice engagement. Thus, Government of Ghana can better succeed with NCDs prevention if there is a clear partnership with the private organization in collaborative approach. Approaching NCDs with a joint-business strategy is deemed efficient due to the complexities of management and prevention of such illness. The success of this partnership can be envisaged when actors agree to work in a multicultural and multifaceted environment.

\section{Acknowledgements}

The author appreciates the invaluable inputs and suggestions from policy makers and research facilitators from the Young Leaders for Health Summit-World Health Assembly organized at Charite University Medical Center, Berlin.

\section{Conflict of interest}

Author declares that there is no conflict of interest.

\section{References}

1. WHO/WEF Report of a Joint Event. Preventing non-communicable disease in the workplace through diet and physical activity. China; 2008:1-52.

2. WHO African Health Monitor. NCDs: an overview of African silent new killers. Africa; 2008:1-60.

3. Global action plans for the prevention and control of non-communicable diseases 2013-2020. USA: WHO; 2013. p. 1-55.

4. MacReady N. UN meeting to address growing burden of noncommunicable diseases. JNCI: Journal of the National Cancer Institute. 2011;103(18):1354-1355.

5. Tenkorang EY, Kuuire VZ. Noncommunicable Diseases in Ghana: Does the Theory of Social Gradient in Health Hold? Health Educ Behav. 2016;43(Suppl 1):25S-36S.

6. Yawson AE, Abuosi AA, Badasu DM, et al. Non-communicable diseases among children in Ghana: health and social concerns of parent/ caregivers. Afr Health Sci. 2016;16(2):378-388.

7. Chinouya MJ, Adeyanju O. A disease called stigma: the experience of stigma among African men with TB diagnosis in London. Public Health. 2017;145:45-50.

8. Kralj V, Cukelj P. Obesity and chronic kidney disease: a public health issue. Cardiologia Croatica. 2017;12(7-8):293-301.

9. Ahenkan A, Aduo-Adjei K. Predictors of Patient Satisfaction With Quality of Healthcare in University Hospitals in Ghana. Hosp Pract Res. 2017;2(1):9-14.

10. Atobrah D. Breast cancer research in Ghana: a focus on social science perspectives. Chronic non-communicable diseases in Ghana: Multidisciplinary perspectives Chapter 4. West Africa; 2013:56-66.

11. Sanuade O,Agyemang C. Stroke in Ghana: A systematic literature review. Chronic non-communicable diseases in Ghana: Multidisciplinary perspectives Chapter 2. West Africa; 2013. p. 29-38.

12. De-Graft Aikins A, Kushitor M, Koram K, et al. Chronic noncommunicable diseases and the challenge of universal health coverage: insights from community-based cardiovascular disease research in urban poor communities in Accra, Ghana. BMC Public Health. 2014;14(Suppl 2):S3. 